\title{
Experimental determination of the onset of turbulence on inclined plates using hot wire velocity measurements
}

\author{
A. A. Rodríguez-Sevillano ${ }^{1}$, I. Pérez-Grande ${ }^{2} \&$ J. Meseguer ${ }^{2}$ \\ ${ }^{1}$ E.U.I.T. Aeronáutica, Universidad Politécnica de Madrid, Spain \\ ${ }^{2} I D R / U P M$, E.T.S.I. Aeronáuticos, \\ Universidad Politécnica de Madrid, Spain
}

\begin{abstract}
The problem of determination of the turbulence onset in natural convection on heated inclined plates in an air environment has been experimentally revisited. The transition has been detected by using hot wire velocity measurements. The onset of turbulence has been considered to take place where velocity fluctuations (measured through turbulence intensity) start to grow. Experiments have shown that the distance to the plate edge where the onset begins depends both on the plate inclination angle and the plate temperature, and thus on the Grashof number defined in terms of the temperature difference between the heated plate and the surrounding air. An experimental setup to measure the above mentioned distance has been developed. In this paper, such an apparatus is presented, as well as the experimental procedure and some experimental results.
\end{abstract}

Keywords: mirror seeing, turbulence onset, natural convection.

\section{Introduction}

One of the problems arising on Earth-based solar telescopes is concerned with image degradation due to the boundary layer, which is formed close to the Sunheated mirror surfaces. Since these surfaces are warmer than the surrounding air, when the surface is inclined the fluid flows over the surface due to convection, and thus a thermal boundary layer is formed. At initial stages the boundary layer flow regime is laminar, but after some distance turbulent eddies are formed and 
the transition to a turbulent boundary layer begins. Further up the flow becomes fully turbulent. Most of the image degradation in telescopes occurs in a thin but very turbulent layer above the surface, such effect being known as mirror seeing, which consists of optical aberrations produced by density non-homogeneities in the air along the optical path. The refraction index of air changes due to thermal non-uniformities so that the wavefront incident on the mirror is randomly distorted, and therefore, images are altered.

Therefore, mirror seeing is the result of natural convection on the mirror surface, for it being warmer than the surrounding air. The effect is generated in the region where the temperature fluctuations are the largest and most intermittent, that is, just above the viscous conductive layer.

Because of its influence in the performance degradation of optical telescopes, the seeing effect has been studied both theoretical and experimentally [1-3].

In order to minimise the mirror seeing effect, the air flow over the mirror has to be laminar, the optical effects being this way small and, what is more important, predictable. Therefore, part of the thermal feasibility study of a telescope should consist of determining whether the convective flow is going to be laminar or turbulent, in this last case being necessary to cool down the mirror to diminish the difference of temperature between mirror and air to keep the laminar regime. It has to be noticed that this mirror seeing effect is much more important in solar telescopes, for being the primary mirror directly facing to and absorbing energy from Sun, what could heat the surface noticeably, hence the interest to determine when the transition from laminar to turbulent in a heated inclined surface occurs, aiming with this information to improve the design, either by avoiding the turbulent regime or at least by predicting the effects and the area it could affect.

Obviously, there are many other thermal related problems in solar telescopes, such as the geometrical deformations due to heating, although in the following only mirror seeing related effect is considered.

The mechanism of natural convection is well understood, however, the complexities of fluid motion make it very difficult to obtain analytical solutions, unless the equations are solved under some simplifying assumptions with the consequent lack of generality. Over the last decades, the transition for inclined plates has been widely studied by several authors [4-7]; a review on the state of the art for the vertical plates can be found in Jannot and Kunc [7].

In this paper, an experimental apparatus designed for the determination of the turbulence onset in natural convection on heated inclined plates in air is presented. The transition has been determined from hot wire velocity measurements. Attention has been focused in the onset of turbulence and not on heat transfer mechanisms. The onset of turbulence has been considered to take place where velocity perturbations (measured through turbulence intensity) start to grow. The amplification of the rms-values of velocity fluctuations has been taken as an indication of the appearance of turbulence.

In the following sections, the experimental setup, the experimental procedure and some experimental results are presented. 


\section{Experimental setup}

The aim of the experiments was to measure air velocity inside the boundary layer of a heated inclined plate by using hot wire anemometry, fig. 1. To fulfil this requirement, an experimental setup allowing air speed measurements at prescribed heights over the heated surface and at different distances from the edge of the heated plate was designed. Both the heated and the measuring equipment must be at different inclinations with respect to the vertical, and both the plate temperature as well as the surrounding one must be controlled.

Taking all the above into account, the developed experimental apparatus (fig.2) consists of a thermal blanket (B) supported by a substratum made of plywood (S), which is hinged to a table (T) in such a way that its tilting angle $\alpha$ can be fixed at any desired value between $0^{\circ}$ and $90^{\circ}$. A translation stage $(\mathrm{G})$ is anchored to the substratum. The linear guide supports an arm (A) which in turn supports the hot-wire probe $(\mathrm{P})$ used to measure air velocity close to the upper surface of the heated surface.

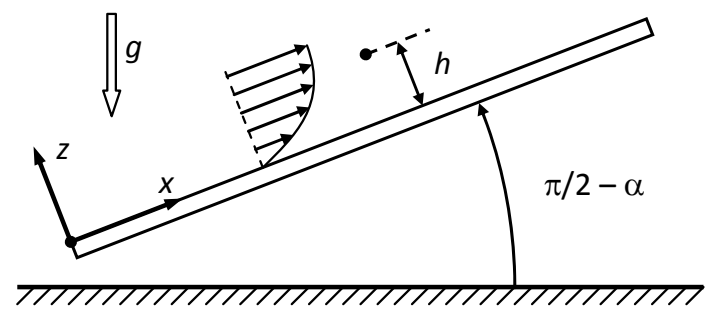

Figure 1: To determine the onset of turbulence in the flow on a heated plate of length $\mathrm{L}_{b}$ inclined at an angle $\alpha$ with respect to the vertical, a hot wire probe is displaced over the plate at a distance $h$.

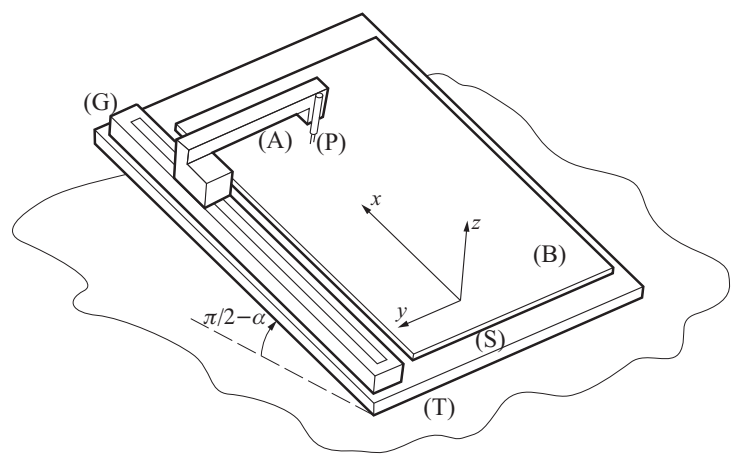

Figure 2: Sketch of the experimental apparatus: thermal blanket (B), substratum (S), table (T), translation stage (G), arm (A), and hotwire probe $(\mathrm{P})$. 


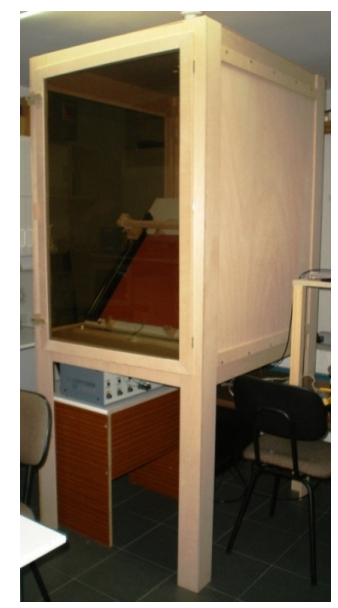

Figure 3: View of the tight test chamber where the apparatus is located.

To avoid undesired perturbations that could force the onset of turbulence on the thermal boundary layer of the plate, the whole described apparatus is placed inside an almost tight chamber whose base is a rectangle $1090 \mathrm{~mm}$ long and 880 $\mathrm{mm}$ wide, being the height of the tight chamber $1190 \mathrm{~mm}$ (fig. 3). The tight chamber is made of wood (a low thermal conductivity material), and it is provided with two doors, one of them placed in the frontal surface of the chamber and the second one in one of the lateral sides. There are five holes 10 $\mathrm{mm}$ in diameter at the ceiling of the tests chamber to allow the pressure equilibrium inside the test chamber.

The translation stage is a TSR-450B model from Zaber, the maximum displacement is $450 \mathrm{~mm}$, the position of the measuring probe being fixed with $\pm 68 \mu \mathrm{m}$ accuracy. Such equipment is placed perpendicular to the bottom side of the blanket, aligned with the $x$-axis, so the probe is displaced parallel to this axis (fig. 1). The lateral position of the probe as well as its vertical distance to the blanket surface can be selected by changing the arm (A), although in the experiments here reported both distances have been kept constant (the prove moves over the mid line of the blanket, at $25 \mathrm{~cm}$ from the lateral sides, and at a distance of $2 \mathrm{~mm}$ over the blanket surface).

The thermal blanket is made of glass-fibre reinforced silicone rubber with an electrical resistance foil embedded. The thermal blanket is squared, $L_{b}=500 \mathrm{~mm}$ side and $0.5 \mathrm{~mm}$ thick, and it can be operated up to a maximum temperature of $530 \mathrm{~K}$. The blanket temperature is controlled through a Watlow PID SD (1/16 DIN) series controller, which allows the blanket temperature $T_{b}$ to be kept between $277 \mathrm{~K}$ and $423 \mathrm{~K}$ with $\pm 0.2 \mathrm{~K}$ temperature stability.

Air temperature inside the tests chamber, $T_{a}$, is measured at $10 \mathrm{~mm}$ over the thermal blanket by using a thermocouple placed close to the top edge of the blanket, at the mid line; this thermocouple is connected to a FLUKE multimeter, model 289. Besides, temperature is also measured at the wall of the tight chamber, $T_{w}$, some $800 \mathrm{~mm}$ over the tight chamber floor, with a standard 
thermometer (this chamber temperature is only used to establish when a steady state is reached in each experimental sequence).

Velocities have been measured by using a Dantec Dynamics 55P0161 hot wire anemometer. The hot wire probe being a Dantec 55P16, equipped with a 5 $\mu \mathrm{m}$ in diameter Platinum-Tungsten wire. The wire probe is parallel to the $y$ axis, so velocities normal to the wire (those contained in the $x z$ plane) are measured. The subsystem for measuring velocities is completed with signal conditioner, an analog-digital converter and a computer equipped with a StreamWare $\mathbb{C}$ software to arrange hot wire anemometer configuration, data acquisition and analysis.

\section{Experimental procedure and results}

Experiments were performed as follows: first of all the desired angle of inclination of the tilting platform $\alpha$ is set, and the hot wire probe is positioned at the lower edge of the blanket at the selected height over the thermal blanket. Then the temperature of the blanket is set to a given value $T_{b}$, and electric power is supplied to the thermal blanket so that the heating of the blanket starts. While thermal blanket is heated, temperatures both close to the blanket surface and at the test chamber wall ( $T_{a}$ and $T_{w}$, respectively) are continuously measured to determine when a steady state is reached at the desired blanket temperature. This steady state is assumed to be reached when recorded temperatures $T_{a}$ and $T_{w}$ do not change with time. It must be pointed out that the time needed to reach such an steady state can be large depending on the difference $\Delta T_{b a}=T_{b}-T_{a}$ between the blanket temperature, $T_{b}$, and the air temperature, $T_{a}$; such a time can be up to two hours for large values of the temperature differences $\Delta T_{b a}$ (that means large values of the blanket temperature).

Velocity measurements start once the steady state is reached. Then the hot wire probe moves along the $x$ axis at $10 \mathrm{~mm}$ steps, and at each measuring position, after waiting for a few seconds to avoid perturbations (if any) due to the probe displacement, velocity is sampled at $10 \mathrm{kHz}$ during $1 \mathrm{~s}$. As already stated the hot wire is placed parallel to the $y$ axis, so that the velocity component in the plane normal to the wire direction is obtained (the plane $x z$ ). Obviously the phenomenon under study depends not only on the distance from the bottom blanket edge to the measuring point, but also on the distance $h$ from the probe to the blanket surface; in this paper only the results corresponding to $h=2 \mathrm{~mm}$ are analyzed, although some additional measurements at $h=10 \mathrm{~mm}$ have been performed, and new measurement campaigns at intermediate distances are envisaged in order to get a better description of the boundary layer development over the blanket.

In order to check the repeatability of the experiment, the above described procedure is carried out at least five times for each pair of values of both the inclination angle $\alpha$ (measured from the vertical) and the blanket temperature $T_{b}$. In general, the different runs within a five-run set were not performed in a unique experimental session, by performing two consecutive runs just one after another, but allowing a gap of several hours between two consecutive runs. 
As explained in the previous section, records of velocities have been obtained for different inclination angles, $\alpha$, and blanket temperatures, $T_{b}$ (note that, as defines in figures 1 and $2 \alpha=0^{\circ}$ means that the plate is vertical). From these data the mean velocity at each position, $U(x)$, as well as the standard deviation or root mean square value, $\sigma(x)$, are calculated, the turbulence intensity being $I_{u}(x)=\sigma(x) / U(x)$.

The results of typical five-run campaigns are shown in figures 4,5 and 6 . These particular results presented to illustrate the measurements correspond to different inclination angles $\alpha$ and blanket temperatures $T_{b}$. In each one of the plots the averaged values of the turbulence intensity obtained from the five different runs performed for the selected values of $\alpha$ and $T_{b}$ are shown, in all cases indication of the scattering of the experimental results is also given. It must be stressed that the sharp leading edge of the thermal blanket was not provided with any faring to smooth it, which could slightly affect the normal development of the boundary layer close to the leading edge, mainly at high values of the inclination angle.
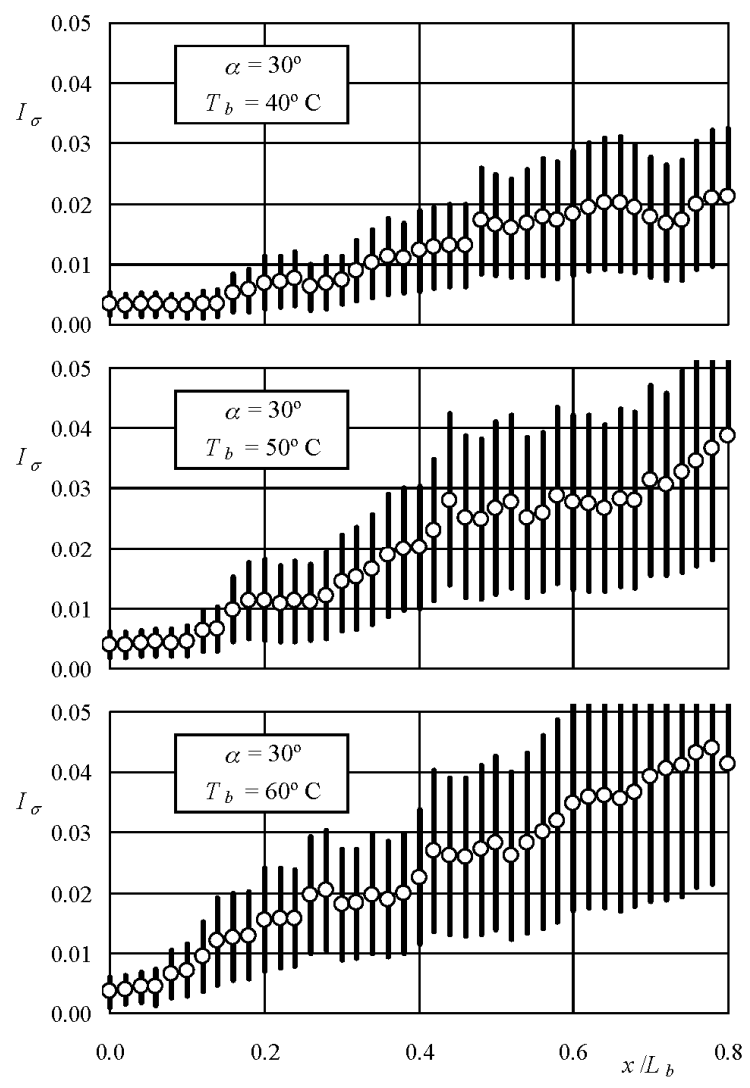

Figure 4: $\quad$ Results for $\alpha=30^{\circ}$. 

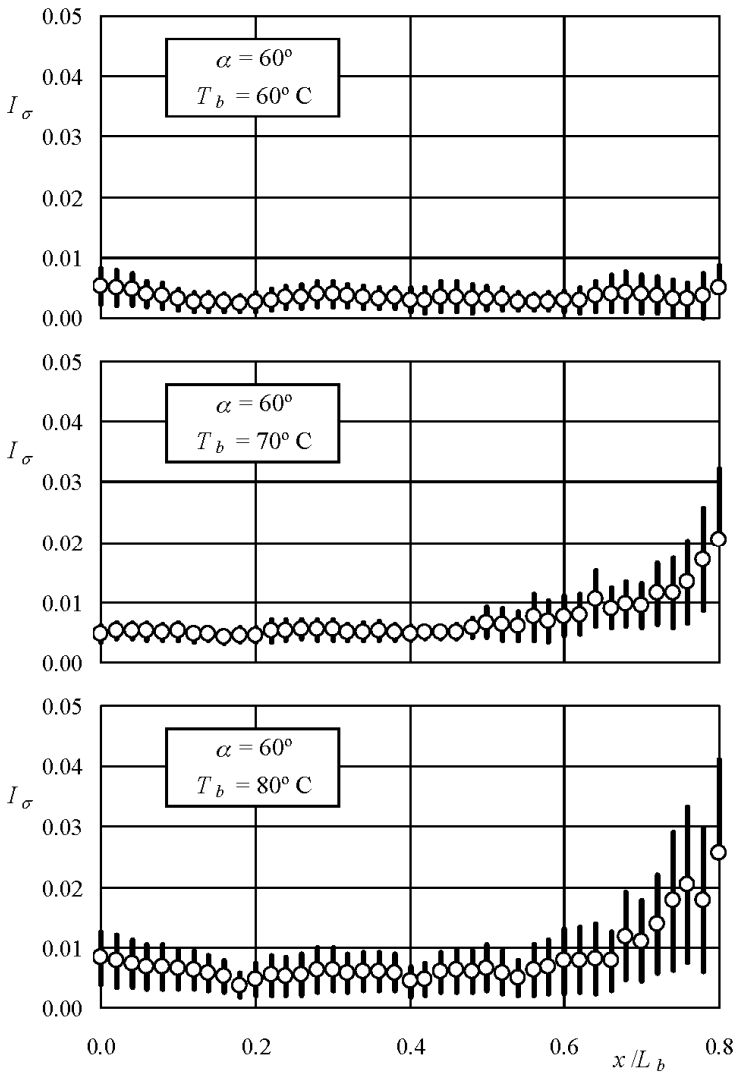

Figure 5: $\quad$ Results for $\alpha=60^{\circ}$.

As it can be observed in the different plots, in spite of the scattering of the experimental data, the results represented in figures 4 to 6 show that close to the bottom edge of the blanket the measured turbulence intensity $I_{u}$ is within a narrow band, the mean value of the turbulence intensity in this region being small and constant or almost constant; after some threshold value of the distance to the bottom edge $I_{u}$ starts to grow, the growing being almost linear at the beginning and much more pronounced at larger distances.

From the different records like the ones presented in figures 4 to 6 , corresponding to the different experimental configurations identified by the parameters $\alpha, T_{b}$, the distance from the leading edge $L\left(\alpha, T_{b}\right)$ where turbulence starts is determined. Indeed, as already mentioned, the determination of the onset of turbulence is not a well defined task, because perturbations start to grow gradually. Nevertheless, in most of the experimental results there is a region close to the blanket leading edge where turbulence intensity is very small and constant or almost constant, and there is another region where turbulence 

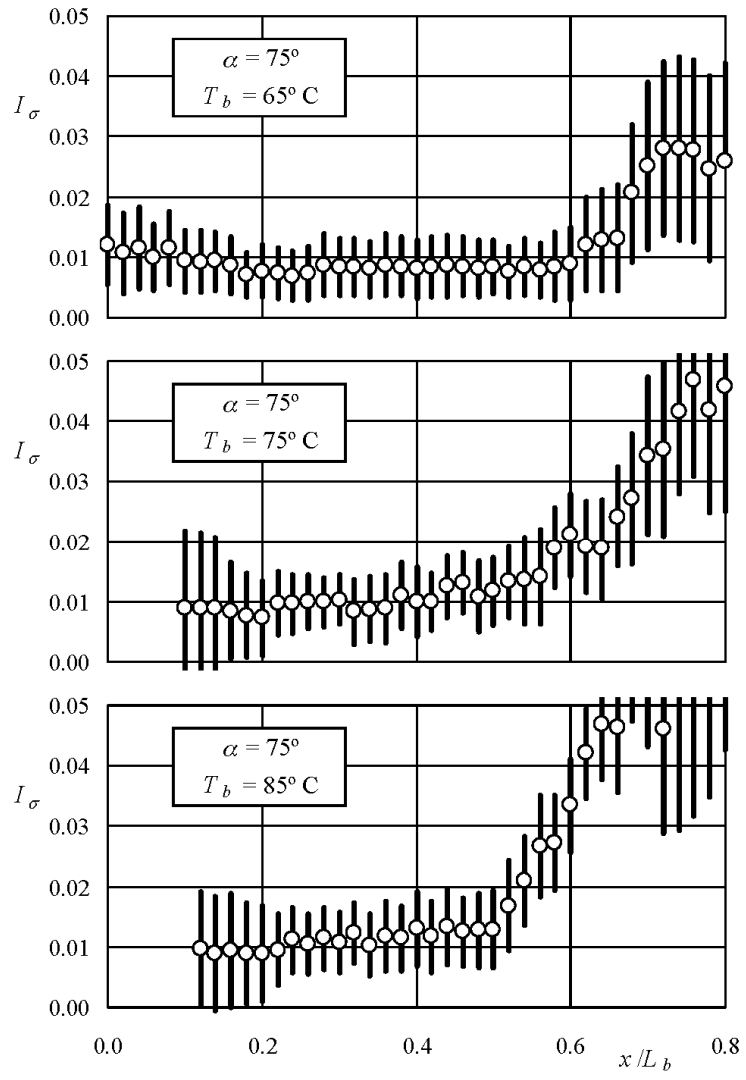

Figure 6: $\quad$ Results for $\alpha=75^{\circ}$.

intensity grows almost linearly. Therefore, the distance from the plate leading edge where these two regions match is defined as the distance $L$ where the onset of turbulence is considered to take place.

Since the driven mechanism of the fluid motion is the difference between blanket and air temperatures, $\Delta T_{b a}=T_{b}-T_{a}$, the variation of the dimensionless distance $L / L_{b}$ with the angle of inclination $\alpha$ and the temperature jump $\Delta T_{b a}$ is summarized in figure 7 . In this plot the length is presented in dimensionless form, the length of the blanket $L_{b}$ being used for this purpose. Obviously, the larger the temperature difference $\Delta T_{b a}$ and the larger the angle $\alpha$ the smaller is the distance $L / L_{b}$ where turbulence starts.

Once determined the distance $L$ where turbulence starts, the Grashof number at this distance $\mathrm{Gr}=\mathrm{g} \beta \Delta T_{b a} L^{3} / v^{2}$ has been calculated; in this expression $\mathrm{g}$ stands for the gravity acceleration $\left(\mathrm{g}=9.81 \mathrm{~m} / \mathrm{s}^{2}\right), \Delta T_{b a}$ is the difference between blanket and air temperatures, $\beta$ stands for the air thermal expansion coefficient, whose variation with temperature, within the interval of temperatures under consideration, is $\beta=\left(3.583-0.0089 T_{m}\right) 10^{-3} \mathrm{~K}^{-1}$, and $v$ is the kinematic viscosity 
Advanced Computational Methods and Experiments in Heat Transfer XI 155

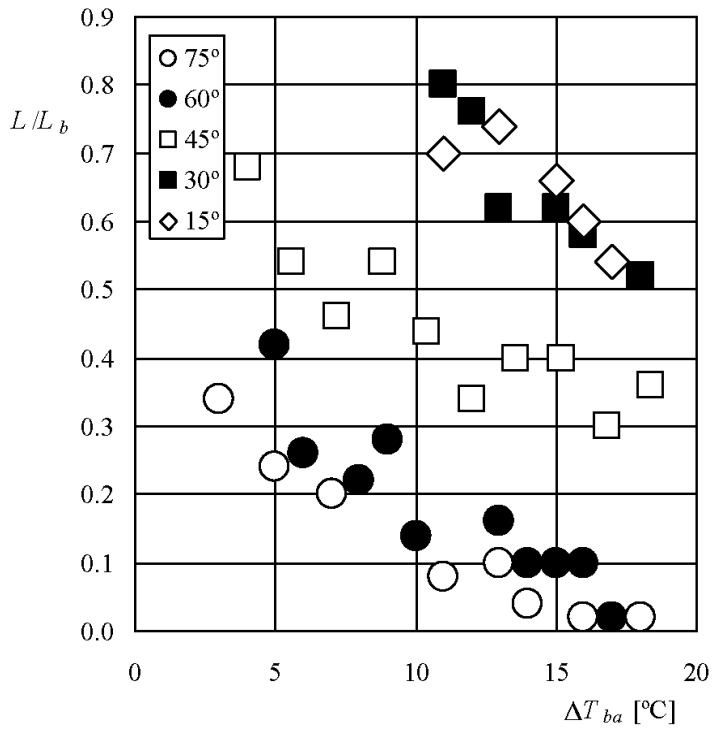

Figure 7: Variation of the dimensionless distance $L / L_{b}$ with the angle of inclination $\alpha$ and the temperature jump $\Delta T_{b a}$.

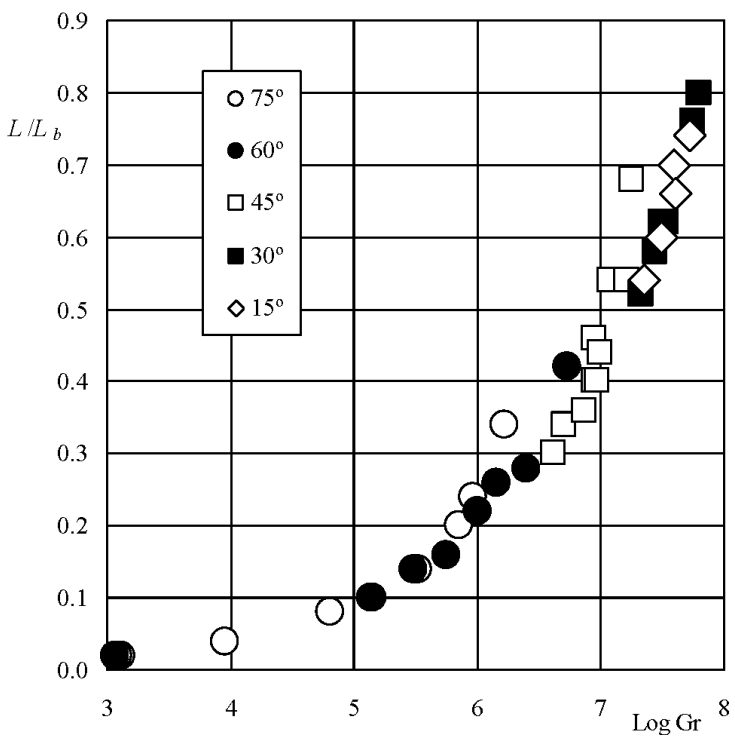

Figure 8: Variation of the dimensionless distance with the Grashof number. 
of air, whose temperature variation within the considered interval of temperatures is $v=\left(1.294+0.01 T_{m}\right) 10^{-5} \mathrm{~m}^{2} / \mathrm{s}$; in these expressions for $\beta$ and $v$ $T_{m}$ is a mean temperature defined as $T_{m}=\left(T_{b}+T_{a}\right) / 2$. The variation of the dimensionless distance with the Grashof number is shown in figure 8 . From this plot it seems that the values of $L / L_{b}$ corresponding to the different blanket inclinations collapse in one single curve when the Grashof number is used instead of the temperature difference $\Delta T_{b a}$.

Although the results shown in fig. 8 are restricted to a separation $h=2 \mathrm{~mm}$ from the blanket surface, they include all the main features of the phenomenon under consideration: there is a region close to the heated surface edge where the flow is laminar, so that seeing effects are negligible in this region. After some distance from the leading edge (whose magnitude depends on the inclination of the plate), flow instabilities start to grow and the flow regime in the boundary layer becomes turbulent, thus causing a noticeable increment of seeing effect.

\section{References}

[1] J.M. Beckers, J. Melnick, Effects of heat sources in the telescope beam on astronomical image quality, SPIE Proc. 2199, pp. 478-480, 1994.

[2] L. Zago, An engineering formulae for local and dome seeing, SPIE Proc. 2871, pp. 726-736, 1996

[3] M.T. Bridgeland, C.R. Jenkins, Measurement of mirror seeing in the laboratory and at the telescope, New Astron. Rev. 42, pp. 435-440, 1998.

[4] D.J. Tritton, Transition to turbulence in the free convection boundary layers on an inclined heated plate, J. Fluid Mech. 16, pp. 417-435, 1963.

[5] G.C. Vliet, Natural convection local heat transfer on constant-heat-flux inclined surfaces, J. Heat Transf.-Trans. ASME, 91, pp. 511-516, 1969.

[6] Y. Jaluria, B. Gebhart, On transition mechanisms in vertical natural convection flow, J. Fluid Mech. 66, pp. 309-337, 1974.

[7] Jannot, T. Kunc, Onset of transition to turbulence in natural convection with gas along a vertical isotherm plane, Int. J. Heat Mass Transf. 41, pp. 43274340, 1998. 